Revue d'histoire de l'Amérique française

REVUE D.HISTOIRE DE L'AMÉRIQUE FRANÇAISE

\title{
Note de recherche : L'aventure de la North West Company en Mauricie, 1799-1814
}

\section{Claude Gélinas}

Volume 53, numéro 3, hiver 2000

URI : https://id.erudit.org/iderudit/005442ar

DOI : https://doi.org/10.7202/005442ar

Aller au sommaire du numéro

Éditeur(s)

Institut d'histoire de l'Amérique française

ISSN

0035-2357 (imprimé)

1492-1383 (numérique)

Découvrir la revue

Citer cette note

Gélinas, C. (2000). Note de recherche : L'aventure de la North West Company en Mauricie, 1799-1814. Revue d'histoire de l'Amérique française, 53(3), 401-419. https://doi.org/10.7202/005442ar
Résumé de l'article

L'histoire des activités commerciales la North West Company dans l'Est du Canada demeure pratiquement inconnue. Pourtant, il existe plusieurs documents d'archives produits par cette compagnie (principalement des livres de comptabilité) qui contiennent des informations sur son commerce dans le Québec-Labrador. À partir d'une analyse de certains de ces livres de comptabilité conservés dans les Archives du Séminaire de Québec, l'auteur brosse les grandes lignes de l'histoire du commerce effectué par la North West Company dans son secteur du Saint-Maurice au Québec.
Tous droits réservés @ Institut d'histoire de l'Amérique française, 2000
Ce document est protégé par la loi sur le droit d'auteur. L'utilisation des services d'Érudit (y compris la reproduction) est assujettie à sa politique d'utilisation que vous pouvez consulter en ligne.

https://apropos.erudit.org/fr/usagers/politique-dutilisation/ 
NOTE DE RECHERCHE

\title{
L'AVENTURE DE LA NORTH WEST COMPANY EN MAURICIE, 1799-1814 ${ }^{1}$
}

\author{
CLAUDE GÉLINAS \\ CÉLAT \\ Université Laval
}

\section{RÉSUMÉ}

L'histoire des activités commerciales la North West Company dans l'Est du Canada demeure pratiquement inconnue. Pourtant, il existe plusieurs documents d'archives produits par cette compagnie (principalement des livres de comptabilité) qui contiennent des informations sur son commerce dans le Québec-Labrador. À partir d'une analyse de certains de ces livres de comptabilité conservés dans les Archives du Séminaire de Québec, l'auteur brosse les grandes lignes de l'histoire du commerce effectué par la North West Company dans son secteur du Saint-Maurice au Québec.

\section{ABSTRACT}

The history of the North West Company presence in Eastern Canada remains practically unknown, even thus many documents produced by the Company (mostly account books) exist and contain information on its commercial activities in the Quebec-Labrador area. Analyzing data furnished by such account books found in the archives of the Quebec Seminary, the author presents a brief survey of the history and commercial activities of the North West Company in its St. Maurice Department, Quebec.

S'il y a une lacune sérieuse dans notre connaissance de l'histoire du commerce des fourrures au Canada, c'est bien le rôle joué par la North West Company dans l'Est du pays. Alors que les activités de cette compagnie dans l'Ouest canadien ont été largement étudiées, on ignore pratiquement tout de ce qu'ont été ses réalisations dans la

1. Cette étude a été rendue possible grâce à l'obtention d'une bourse d'études postdoctorales du Conseil de recherches en sciences humaines du Canada (CRSH) et d'une bourse postdoctorale conjointe du CÉLAT (Université Laval) et du Musée de la civilisation (Québec). Je tiens à remercier tout particulièrement Andrée Gendreau et Hélène Dionne pour leur appui lors de mon séjour au Musée de la civilisation, de même que Danielle Aubin et le personnel du Musée d'histoire de l'Amérique française qui ont grandement facilité mes recherches dans les archives du Séminaire de Québec.

RHAF, vol. 53, $\mathrm{n}^{\circ} 3$, hiver 2000 
péninsule Québec-Labrador. Ce qu'on sait, c'est qu'au moment de son apogée, entre 1800 et 1810 , la North West Company était présente dans le bassin hydrographique de la baie James, en Abitibi et au Témiscamingue, qu'elle contrôlait le commerce des fourrures dans les bassins de l'Outaouais et du Saint-Maurice, en plus d'être locataire des King's Posts et de la seigneurie de Mingan. En fait, la compagnie occupait tout l'intérieur des terres où la Hudson's Bay Company était absente. Cette position stratégique lui permettait ainsi d'intercepter d'importantes quantités de fourrures qui, normalement, auraient dû gagner les postes de la compagnie rivale sur la côte jamésienne. Mais au-delà de ces grandes lignes, on ignore l'essentiel de ses activités commerciales dans l'Est du Canada.

Parmi les facteurs responsables de cette carence, il y a le fait que la grande majorité des archives de la North West Company qui nous sont connues concernent les activités de la compagnie dans l'Ouest canadien ${ }^{2}$. Par ailleurs, contrairement aux archives de la Hudson's Bay Company, celles de la North West Company n'étaient pas centralisées systématiquement en un même endroit, de sorte qu'elles sont, aujourd'hui, dispersées dans divers dépôts d'archives. Au Québec seulement, on trouve certains de ces documents originaux, notamment, dans les archives du musée McCord, dans les archives de l'Université de Montréal, dans celles des Sulpiciens et de la ville de Montréal, ainsi qu'aux Archives nationales du Québec. Et il ne fait aucun doute que d'autres documents de la North West Company dorment dans des dépôts d'archives moins connus, et que certains d'entre eux pourraient renfermer des informations sur les opérations de la compagnie au Québec. On en veut pour preuve l'exemple des Archives du Séminaire de Québec ${ }^{3}$.

En effet, les archives du Séminaire de Québec contiennent une vingtaine de livres de comptabilité et de correspondance ayant appartenu à la North West Company et qui, au total, font plus de 2500 pages $^{4}$. Un des principaux intérêts de cette collection est que plusieurs de

2. Soulignons toutefois que les archives de la Hudson's Bay Company à Winnipeg et Ottawa comptent plus d'une quinzaine de livres de comptabilité ayant appartenu à la North West Company et qui sont reliés aux activités commerciales de celle-ci en territoire québécois (F. 462).

3. Depuis 1995, les archives du Séminaire de Québec sont gérées par le Musée d'histoire de 1'Amérique française (Musée de la civilisation, Québec).

4. Une partie de cette documentation a déjà été utilisée par certains chercheurs, mais dans le cadre d'études qui ne portaient pas spécifiquement sur l'histoire de la North West Company au Québec. Harold A. Innis, «The North West Company », Canadian Historical Review, 8 (1927): 316; W. K. Lamb, The Journals and Letters of Sir Alexander Mackenzie (London, Cambridge University Press, 1970). Par ailleurs, le parcours historique de ces documents d'archives reste à être établi. Il semble assez évident qu'ils appartenaient presque tous à 
ces documents renferment des informations textuelles ou statistiques sur les activités commerciales de la compagnie dans ses secteurs des King's Posts, du Saint-Maurice, de Grand River (Outaouais) et du Témiscamingue entre 1800 et $1821^{5}$. Tandis que les livres de correspondance nous renseignent sur le personnel de la compagnie dans les différents secteurs québécois et sur la localisation des postes de traite, les livres de comptabilité fournissent des chiffres révélateurs sur la rentabilité du commerce dans les divers postes, sur l'évolution du prix des articles de traite et des fourrures, sur la nature des fourrures obtenues, etc. Bref, ce corpus d'archives constitue une source d'information d'une grande valeur pour documenter l'histoire de la compagnie en sol québécois ${ }^{6}$. Afin de faire ressortir le potentiel de cette documentation, je présenterai ici une brève analyse des données qui concernent plus particulièrement les opérations de la North West Company dans le St. Maurice Department entre 1799 et 1814.

la Sir Alexander McKenzie \& Co. - connue également sous les noms de XY Company ou de New North West Company — qui fut fondée en 1801 avant d'être rattachée à la North West Company à l'hiver de 1804-1805. L.-R. Masson, Les Bourgeois de la Compagnie du Nord-Ouest (New York, Antiquarian Press, 1960), 1: 1889-1890, : 76-77, 85 ; Russell A. Pendergast, The XY Company, 1798-1804, thèse de doctorat, Université d'Ottawa, 1957). Toutefois, on ignore dans quelles circonstances ils sont passés des bureaux montréalais de la compagnie aux archives du Séminaire de Québec. L'abbé Henri-Raymond Casgrain (1831-1904) a peut-être été celui qui en a fait l'acquisition dans la seconde moitié du XIX ${ }^{\mathrm{e}}$ siècle. Marie-Josée Courchesne et Chantal Aubin, Plus de trois siècles d'histoire à découvrir. Les archives du Séminaire de Québec (Québec, Musée de la civilisation, Service des archives et de la documentation, Direction de la recherche et de la conservation, 1998), 106-107. Certains de ces documents ont été microfilmés et peuvent être consultés aux Archives nationales du Canada (Sir Alexander MacKenzie Papers, MG 19, M1349-1351, volumes 1-20).

5. Les documents pertinents pour l'étude du commerce de la North West Company au Québec sont, selon le système de cotation actuel des manuscrits: 0-516, 0-520, 0-521, 0-523, 0-524 (en particulier), 0-526 et 0-531. Les autres documents qui ne concernent pas directement les opérations de la compagnie au Québec consistent en des livres de comptes personnels d'employés et de dirigeants (0-522 et 0-527), un résumé du partage des revenus entre les «partenaires » (0-525), un inventaire des marchandises expédiées dans l'Ouest (0-517) et divers livres de comptabilité générale (0-528, 0-529, 0-530 et 0-532 à 0-537).

6. Sur le potentiel des livres de comptabilité des compagnies de traite pour les études historiques, voir notamment Arthur J. Ray, «The Early Hudson's Bay Company Account Books as Sources for Historical Research : An Analysis and Assessment», Archivaria, 1,1 (1975-1976); «The Hudson's Bay Company Account Books as Sources for Comparative Economic Analyses of the Fur Trade: An Examination of Exchange Rate Data», Western Canadian Journal of Anthropology, 6,1 (1976); Arthur J. Ray et Donald B. Freeman, " "Give Us Good Measure" ": An Economic Analysis of Relations Between the Indians and the Hudson's Bay Company Before $1763 »$ (Toronto, University of Toronto Press, 1978); Claude Gélinas, «La traite des fourrures en Haute-Mauricie avant 1831. Concurrence, stratégies commerciales et petits profits ", Revue d'histoire de l'Amérique française, 51,3 (1998); Russell A. Pendergast, op. cit. 


\section{LA NORTH WEST COMPANY EN MAURICIE}

La création de la North West Company fut un corollaire de la nouvelle conjoncture économique dans laquelle s'inscrivait le commerce des fourrures au lendemain de la conquête anglaise. Si on ignore la date exacte de la fondation officielle de cette compagnie bien que certaines sources laissent entendre 1776 ou encore 1779 - , il est acquis qu'elle existait en bonne et due forme au tout début des années $1780^{7}$. Concrètement, il s'agissait d'une association de marchands qui, devant la montée croissante des coûts d'exploitation - il fallait notamment se rendre de plus en plus loin dans les régions des Grands Lacs et du Nord-Ouest pour obtenir des fourrures avaient jugé bon de mettre en commun leurs capitaux respectifs et de se partager les revenus de la traite au pourcentage de leurs investissements ${ }^{8}$. Il s'agissait d'une structure organisationnelle bien différente de celle de la Hudson's Bay Company qui constituait alors une compagnie à charte jouissant, théoriquement, d'un droit exclusif d'exploitation sur l'ensemble du bassin hydrographique de la baie d'Hudson. Jusque dans les années 1810, la North West Company connut une grande prospérité grâce au véritable monopole commercial qu'elle avait réussi à établir autour de la Terre de Rupert. Les petits commerçants indépendants, incapables entre autres de concurrencer les prix offerts par la North West Company, n'avaient souvent d'autre choix que de joindre ses rangs.

On peut croire que l'avènement de la North West Company en Mauricie a résulté d'une telle absorption de petits commerçants indépendants locaux, bien que le fil exact des événements demeure difficile à documenter. En effet, on ne connaît pas précisément en quelle année et de quelle façon la North West Company s'est implantée dans la région. Rien n'indique que les frères Giasson, qui exploitèrent des postes de traite à la Rivière-aux-Rats et à Weymontachie entre 1778 et 1780 , fussent rattachés à la compagnie ${ }^{9}$, de sorte que l'arrivée de celle-ci en Mauricie dut se faire après 1780. En 1786, trois licences auraient été octroyées à des commerçants indépendants pour la traite sur le Saint-Maurice, mais rien n'indique, une fois de plus, que ceux-

7. W. Stewart Wallace, dir., Documents Relating to the North West Company (Toronto, The Champlain Society, 1934), 5-6.

8. Gordon C. Davidson, The North West Company (New York, Russell \& Russell, 1967 [1918]), 15. Ann Carlos, The North American Fur Trade, 1804-1821: A Study in the Life Cycle of a Duopoly (New York/London, Garland, 1986).

9. Claude Gélinas, Haldimand, Godefroy de Tonnancour et les Têtes de Boule: commerce des fourrures et société autochtone en Haute-Mauricie dans les années 1770. Communication présentée au colloque Nord-Laval en sciences humaines, Université Laval, 18-19 mars 1999. 
BASSIN HYDROGRAPHIQUE DE LA RIVIÈRE SAINT-MAURICE

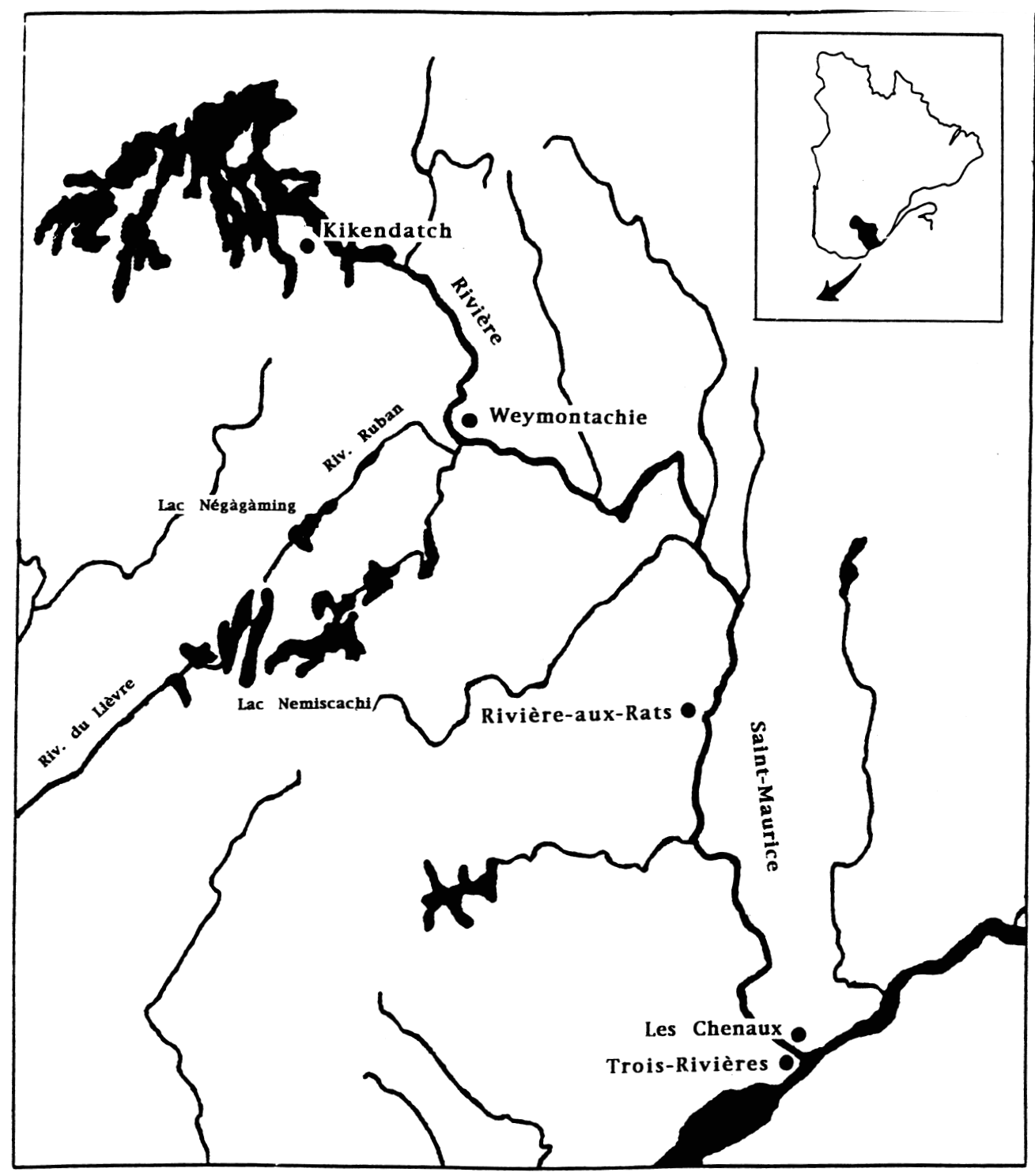

Source: l'auteur. 
ci aient été rattachés à la North West Company ${ }^{10}$. Enfin, en 1798, Claude Pratte, un des commerçants qui travaillaient alors dans le Haut-Saint-Maurice, ne semblait pas non plus associé à la compagnie; il semblait plutôt collaborer avec le marchand Charles-Antoine Godefroy de Tonnancour ${ }^{11}$. Toutefois, en 1800, ce même Pratte fit adresser une lettre aux dirigeants de la North West Company les informant qu'ils devraient rembourser le marchand Hart de Trois-Rivières pour la somme de $398 £$ pour l'achat de marchandises de traite ${ }^{12}$. Aussi, puisque, jusqu'en 1798, Pratte s'approvisionnait lui-même chaque printemps auprès de Hart qu'il remboursait en fourrures à son retour des Hauts-mauriciens en juillet, et puisque, en 1800, le remboursement du marchand Hart semblait désormais s'effectuer par l'entremise de la North West Company, il se pourrait que celle-ci ait absorbé Pratte, et peut-être d'autres commerçants mauriciens, en 1799 ou en $1800^{13}$. Cependant, à ma connaissance, aucun document d'archives de la compagnie ne fait état d'activités commerciales en Haute-Mauricie à cette époque.

En 1802, l'année même où elle obtenait la location des King's Posts, la North West Company aurait possédé trois postes le long de la rivière Saint-Maurice ${ }^{14}$. C'est d'ailleurs à cette même date que l'on trouve, dans les livres de comptabilité de la compagnie, les premières références à $l^{\prime}$ «aventure du Saint-Maurice ${ }^{15}$ ». Un de ces postes mauriciens était vraisemblablement situé à la Rivière-aux-Rats ${ }^{16}$, et tout indique qu'il fut exploité jusqu'à la cessation des activités de la com-

10. Gordon C. Davidson, op. cit., 26.

11. Archives du Séminaire de Trois-Rivières (ASTR), Fonds Aaron Hart, 1797.

12. Ibid., 1800 .

13. En fait, en mars 1799, Alexander McKenzie, qui pourtant à cette époque «does not [...] form a very favorable opinion of the St. Maurice trade» avait entrepris en vain des démarches pour étendre les activités de la North West Company en Mauricie. Frobisher to McTavish, 16 mars 1799, Hudson's Bay Company Archives (HBCA), F 3/1, North West Company Correspondence, 1791-1799, fo. 314. Or, au même moment, Pratte cherchait à conclure une entente avec la compagnie: «Prate [...] was up here [Montréal] some time in Feby. to dispose of his property in land to us for which he expected about $£ 1000$ », de sorte que, effectivement, la saison 1799-1800, ou encore le printemps de 1800, pourrait marquer le début des opérations de la North West Company en sol mauricien. McGillivray to McTavish, 23 mars 1799, Ibid., fo. 316 .

14. Gordon C. Davidson, op. cit., 87-88.

15. North West Company Account Book, 1799-1804, 30 septembre et 2 octobre 1802. Archives du Séminaire de Québec (ASQ), collection des manuscrits, 0-521. Une de ces références semble indiquer que la compagnie engagea des Iroquois, à l'automne de 1802, pour transporter de la marchandise en Haute-Mauricie: «an Indian for coming to town [Montréal] from St. Registe about the Adv[entu]re to the St. Maurice».

16. Louis-P. Cormier, Jean-Baptiste Perrault marchand voyageur parti de Montréal le $28^{e}$ de mai 1783 (Montréal, Boréal Express, 1978), 104; HBCA, F 4/2, North West Company Account Book, 1806-1807 [Rat River]. 
pagnie en Mauricie. Toujours en 1802, dans une lettre adressée à l'agent Sutherland à Montréal, un nommé François Albert, de la Rivière-du-Loup (Louiseville), offrait ses services à la North West Company comme commis, soit «aux Chenaux ${ }^{17}{ }^{1}$ sur la rivière Saint-Maurice, soit pour «le haut de la rivière du Lièvre ${ }^{18} »$. Ainsi, il y aurait eu un second établissement de la North West Company situé tout près de Trois-Rivières, et c'était sans doute celui indiqué sur la carte du marchand Jean-Baptiste Perrault, sur la rive est du Saint-Maurice, à quelques kilomètres en amont de la ville ${ }^{19}$. Enfin, on ne dispose d'aucune information précise au sujet de l'emplacement du troisième poste; il aurait pu s'agir du «fort Black» situé au lac Négàgàming sur la rivière Ruban $^{20}$, ou encore d'un poste situé au "portage à Parent», à la hauteur des terres qui séparent les bassins du Saint-Maurice et du lac Saint-Jean ${ }^{21}$. Au total, vers 1802, les trois postes de la North West Company en Mauricie employaient quatorze individus : cinq commis et interprètes, et neuf journaliers ${ }^{22}$.

En somme, tout ce qu'on peut conclure pour l'instant, c'est que la North West Company s'est vraisemblablement implantée en Mauricie quelque part entre 1799 et 1802 , peut-être après avoir absorbé le ou les commerçants locaux. D'ailleurs, à cette époque, la compagnie était entrée dans une compétition féroce avec divers concurrents commerciaux, à savoir la Hudson's Bay Company, la Sir Alexander McKenzie \& Co., à compter de 1801, et d'autres commerçants indépendants. Si les dirigeants de la North West Company prirent la décision d'absorber les commerçants de la Mauricie avant 1802, leur but aurait pu être alors de faire concurrence aux locataires des King's Posts à partir de la Haute-Mauricie et, peut-être aussi, dans une moindre mesure, à la Hudson's Bay Company ${ }^{23}$. Par contre, si la décision avait été prise en 1802, elle se serait alors inscrite dans un mouvement expansionniste bien documenté de la North West Company ${ }^{24}$ et qui, dans l'Est canadien, s'est notamment traduit par l'obtention du bail des King's Posts

17. L'expression «les Chenaux» fait référence aux trois branches de la rivière SaintMaurice à sa confluence avec le fleuve Saint-Laurent. Armour Landry, Bribes d'histoire (TroisRivières, Éditions du Bien Public, 1933), 14.

18. Lettre de Hart à Sutherland, 29 novembre 1802 et lettre de Sutherland à Mailloux, 31 janvier 1803, North West Company Correspondence Book, 1802-1809. ASQ, collection des manuscrits, 0-526. Plus précisément, il pourrait être question ici du lac Némiscachi où la North West Company exploitait un poste en 1806-1807. HBCA, ibid., fo. 20.

19. Louis-P. Cormier, op. cit., 106.

20. Ibid., 107.

21. Ibid.

22. Gordon C. Davidson, op. cit., 280.

23. Claude Gélinas, «La traite des fourrures... », loc. cit., 397-399.

24. L.-R. Masson, op. cit., 1: 77-80. 
et par des tentatives d'implantation dans le bassin hydrographique de l'est de la baie James ${ }^{25}$.

En 1806, Alexander Fraser, stationné à la Rivière-aux-Rats, était directeur du St. Maurice Department pour la North West Company, tandis que Jean-Baptiste Perrault était en charge des postes situés en Haute-Mauricie $^{26}$. En juillet de la même année, celui-ci construisit un poste à Weymontachie et il envoya au lac Négàgàming un nommé Black chargé de faire la traite avec les Algonquins du Lac-des-DeuxMontagnes venus chasser dans le secteur ${ }^{27}$. Perrault fut remplacé l'année suivante par un certain Morrison ${ }^{28}$ et, outre les noms de certains employés, on sait peu de choses sur la présence et les activités de la North West Company en Mauricie par la suite, sinon que son établissement de Weymontachie était sous la gouverne d'un certain Jos Godin en $1812^{29}$.

Par ailleurs, à compter de 1814, la North West Company arrivait désormais difficilement à gérer la hausse des coûts d'exploitation et à résister aux pressions exercées par la Hudson's Bay Company. Elle commença à accumuler des déficits et, aux yeux de plusieurs de ses dirigeants, il devenait inévitable que la sauvegarde de leurs intérêts économiques passait par une union avec la Hudson's Bay Company. C'est ainsi qu'en 1821, la North West Company fut finalement absorbée par la compagnie anglaise, ce qui devait ouvrir une nouvelle ère dans le commerce des fourrures sur le territoire canadien. Toutefois, il est possible que les «Nor'Westers» n'aient pas attendu la fusion pour se retirer de la Mauricie. En effet, les archives de la North West Company ne livrent plus aucun indice d'activités commerciales dans cette région après $1814^{30}$. D'ailleurs, il est envisageable qu'en raison des difficultés financières qu'ils éprouvaient à cette époque, les dirigeants de la compagnie aient fait le choix d'abandonner un secteur

25. W. Stewart Wallace, «The Nor'Westers Invade the Bay », The Beaver, 277 (1947); K. G. Davies, dir., Northern Quebec and Labrador Journals and Correspondence, 1819-1835 (London, The Hudson's Bay Company Record Society, 1963), 293, 299-300, 318.

26. Louis-P. Cormier, op. cit., 103-105.

27. Ibid., 105, 107.

28. Ibid., 108

29. North West Company Account Book, 1804-1813. ASQ, collection des manuscrits, 0-524, 492; Archives nationales du Canada (ANC), Indian Affairs, Miscellaneous Petitions, Correspondence, Regulations, Commissions, 1811-1815. RG-10, vol. 627, 182909-182912.

30. Voir notamment North West Company Account Book, 1804-1813, Ibid.; HBCA, F. 3/1-9, 10, 14, 17, 33, 43, 54, 57. C'est ce qui expliquerait, entre autres, qu'il ne soit aucunement fait mention de postes de traite en Mauricie dans le recensement des établissements de la North West Company et de la Hudson's Bay Company au moment de la fusion en 1821. HBCA, A. 64/26, List of Hudson's Bay Company and North West Company Posts, 1820-1821. 
qui, comme il en sera maintenant question, leur coûtait plus qu'il ne leur rapportait ${ }^{31}$.

\section{LES CARACTÉRISTIQUES DU COMMERCE EN MAURICIE}

L'analyse des statistiques relatives au commerce effectué par la North West Company en Mauricie révèle que le St. Maurice Department était de loin le moins productif des secteurs de la compagnie dans l'Est canadien. Cette situation allait d'ailleurs s'accentuer à compter de 1807, lorsque, comme il sera question plus loin, les postes de la compagnie en Mauricie devinrent des instruments davantage stratégiques que commerciaux. De même, en 1805-1806, la première année pour laquelle on dispose de chiffres, les revenus - il faut entendre ici par revenus la valeur des fourrures recueillies dans les postes de traite - tirés du St. Maurice Department se chiffraient à $2654 £$, une somme nettement inférieure à celle des autres postes circonvoisins, à savoir ceux de Grand River, du Témiscamingue et des King's Posts (tableau 1). La saison suivante, les revenus furent en hausse, passant à $3479 £$. Mais à compter de 1807-1808, ils connurent une tendance générale à la baisse. Cette année-là, ils étaient redescendus à $2453 £$, pour baisser encore à $2021 £$ en $1808-1809$ et à $1146 £$ en 1809-1810. Et après avoir connu une légère hausse en 1810-1811 $(1932 £)$ et $1811-1812(1419 £)$, les revenus glissèrent à seulement $560 £$ à la fin de la saison $1812-1813^{32}$. Les postes mauriciens ne généraient plus alors que $1,9 \%$ des revenus provenant du territoire québécois, comparativement à 5,5\% en 1805-1806.

Comme l'indique le tableau 1, la diminution des revenus annuels fut commune à l'ensemble des secteurs de la North West Company au Québec durant cette période, et on s'explique encore mal cette situation. Il serait surprenant qu'un tel phénomène découle d'une compétition plus féroce de la part de la Hudson's Bay Company, puisque celle-ci n'a pas véritablement réussi à développer des activités commerciales stables à l'intérieur du Québec-Labrador avant la fin des

31. Il ne faudrait pas écarter la possibilité qu'à compter de 1814 , les postes de Mauricie aient été transformés en avant-postes d'établissements plus importants comme les King's Posts ou le poste du Lac des Sables et que, par conséquent, les statistiques relatives au commerce qui s'y serait déroulé, peut-être jusqu'en 1821, aient été fondues dans celles de ces derniers postes. Toutefois, il n'existe actuellement aucun indice pour appuyer un tel scénario.

32. North West Company Account Book, 1804-1813, fos. 34, 98, 197, 235, 295, 387, $427,492$. 
TABLEAU 1

ÉVOLUTION DES REVENUS, EN LIVRES ET EN POURCENTAGE DANS LES SECTEURS DE LA NORTH WEST COMPANY AU QUÉBEC 1805-1813

1805-1806

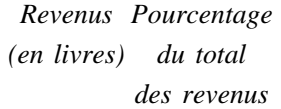

King's Posts Témiscamingue Grand River St. Maurice Total

53,2
31,6
9,7
5,5

2654

47914

\section{9-1810}

Revenus Pourcentage

(en livres) du total

des revenus

19995

12144

2564

1146

35849

55,8

33,9

7,2
1812-1813

Revenus Pourcentage

(en livres) du total

17755

des revenus

9066

59,2

2620

30,2
3,2

560

30001

Source : ASQ, North West Company Account Book, 1804-1813.

années $1810^{33}$, ou de la part de petits commerçants indépendants. D'ailleurs, un passage des Selkirk Papers, cité par Davidson, souligne qu'en 1810: "On Lake Superior, Nipisying, the Gran River and St. Maurice, tho' the trade is by law open to all his Majys. Subjects, the N.W.Co. have no rivals ${ }^{34}$. Il se peut que le blocus napoléonien, officiellement amorcé en 1806, et qui priva tant la Hudson's Bay Company que la North West Company de leur important marché anglais, ait amené cette dernière à diminuer l'intensité de son commerce ${ }^{35}$. Par contre, cette situation fut de courte durée et ne saurait expliquer le déclin prolongé des revenus, d'autant plus que la North West Company fut moins affectée que la Hudson's Bay Company par cet obstacle, puisqu'elle pouvait diversifier ses marchés. Enfin, il y aurait eu une diminution significative des populations d'animaux à fourrures dans l'Ouest canadien à compter de $1810^{36}$, mais on ignore

33. K. G. Davies, op. cit., 288-326; Daniel Francis et Toby Morantz, Partners in Furs. A History of the Fur Trade in Eastern James Bay, 1600-1870 (Kingston et Montréal, McGillQueen's University Press, 1983), 98-115.

34. Gordon C. Davidson, op. cit., 169.

35. Ann Carlos, «The Causes and Origins of the North American Fur Trade Rivalry: 1804-1810», Journal of Economic History, 41 (1981) : 781-784.

36. Ann Carlos, «The Birth and Death of Predatory Competition in the North American Fur Trade : 1810-1821», Explorations in Economic History, 19 (1982): 165-167. 
si une telle diminution a également prévalu au Québec-Labrador, ce qui aurait pu contribuer à réduire la quantité des entrées de fourrures dans les postes de la North West Company.

Quoi qu'il en soit, il reste que la diminution des revenus fut beaucoup plus significative dans le St. Maurice Department qu'ailleurs. Entre 1806 et 1813, ses revenus diminuèrent de 78,9\%, comparativement à 43,6\% pour ceux du Grand River Department, à 40,1\% pour ceux du Temiscamingue Department et à $30,3 \%$ pour ceux des King's Posts (tableau 1). Tout porte à croire que cette situation particulière en Mauricie a découlé de la décision des dirigeants de la North West Company de modifier le rôle de leurs postes de traite de cette région. Un indice de cela se trouve dans le procès-verbal d'une assemblée tenue par ces mêmes dirigeants à Fort William en 1807: «A long conversation then followed relative to the St Maurice \& Kings Posts Departments, and the Wintering partners unanimously desired that the Outfit of the former should be in future so limited as to secure the Concern from loss, - that River being considered merely as a barrier to defend the borders of the neighbouring posts, and the Outfit thereto to be adapted to this purpose only ${ }^{37}$.»

Une telle initiative, qui peut être interprétée comme une façon de ne pas encourager les autochtones à commercer outre mesure dans les postes de la Mauricie, pour du même coup favoriser la fréquentation des postes du Domaine, pourrait se refléter dans le système de crédit en vigueur dans la région. En effet, le crédit était déjà une institution bien implantée en Mauricie à l'arrivée de la North West Company. Si bien qu'en 1806, Perrault fit crédit aux autochtones dès son arrivée à Weymontachie, alors que ses hommes étaient encore à bûcher le bois qui allait servir à la construction du poste ${ }^{38}$. Or, en 1808 , la North West Company stipulait, dans sa politique de crédit, que «The Credits due at Temiskamingue not to exceed in value Twelve hundred Pounds Currency - Those of the other posts of the lower Departments, abating the King's Posts, we consider good for nothing ${ }^{39}[\ldots] »$ Cette réticence à accorder des crédits aux autochtones constituait peut-être un autre moyen d'éloigner ceux-ci des postes mauriciens.

37. W. Stewart Wallace, dir., op. cit., 248.

38. Louis-P. Cormier, op. cit., 105. Dès les années 1760, certains chasseurs autochtones de la Haute-Mauricie avaient l'habitude d'obtenir des crédits auprès des marchands qu'ils venaient rencontrer à Trois-Rivières, et les premiers commerçants qui se sont établis dans les Hauts-mauriciens, à la fin des années 1770, avaient également l'habitude de faire crédit aux chasseurs locaux. Claude Gélinas, Haldimand..., op. cit.

39. W. Stewart Wallace, dir., op. cit., 251-252. 
En fait, comme je l'ai souligné ailleurs, la plupart des postes de traite érigés en Haute-Mauricie, à compter de la fin du XVIII ${ }^{\mathrm{e}}$ siècle, semblaient avoir pour principal objectif de faire concurrence aux King's Posts du bassin du lac Saint-Jean ${ }^{40}$. Or, en devenant locataire des King's Posts en 1802, la North West Company faisait elle-même concurrence à ses postes du Domaine en exploitant à plein régime ses établissements de la Haute-Mauricie. C'est pourquoi, à compter de 1807, ceux-ci furent recyclés en simples lieux de surveillance destinés à prévenir les incursions dans les terres du Domaine des commerçants et des chasseurs de la région de Trois-Rivières. Dès lors, on peut présumer que, face à une réduction probable des inventaires dans les postes de la Mauricie, les autochtones de la région intensifièrent le commerce qu'ils avaient d'ailleurs l'habitude de faire non seulement dans les King's Posts, mais également dans les postes de la Hudson's Bay Company à la baie James et ce, directement ou par voie d'intermédiaires ${ }^{41}$. Un autre indice qui pourrait confirmer une diminution des inventaires est le fait que les entrées de fourrures dans les postes de la Mauricie ont diminué, même si la North West Company n'a pas réduit les prix offerts pour les peaux. Au contraire, le tableau 2 montre que le prix des peaux de castor et de rat musqué a connu une augmentation constante entre 1805 et 1810 . Si, durant la même période, les prix des peaux de loutre, de martre et de vison ont légèrement diminué, il s'est agi d'une baisse de prix qui fut généralisée à l'ensemble des postes québécois ${ }^{42}$.

Bref, même si les autochtones de la Mauricie et de sa périphérie pouvaient encore obtenir, grosso modo, les mêmes prix pour leurs fourrures dans les postes de la région après 1807, il n'y avait sans doute plus suffisamment d'articles de traite ni de possibilité d'obtenir de bons crédits à ces établissements pour maintenir une clientèle assidue, ce qui expliquerait la diminution plus prononcée qu'ailleurs des entrées de fourrures (tableau 3), et par conséquent des revenus dans le St. Maurice Department.

Néanmoins, il se peut que, jusqu'en 1807, la North West Company ait réussi à générer des profits dans son commerce en Mauricie. En effet, si on considère qu'en 1866-1867, au cœur des années de grande prospérité de la Hudson's Bay Company en Mauricie, celle-ci avait recueilli 57 paquets de fourrures ${ }^{43}$, la récolte de 47 paquets par la North West Company, en 1805-1806, paraît plus que respectable.

40. Claude Gélinas, «La traite des fourrures... », loc. cit., 398-399.

41. Claude Gélinas, Haldimand..., op. cit.

42. ASQ, op. cit. (1804-1813).

43. HBCA, B. 230/a/5, fo. 23, Weymontachingue Post Journal, 1866-1868. 
TABLEAU 2

\section{ÉVOLUTION DES PRIX* OFFERTS POUR LES FOURRURES PAR LA NORTH WEST COMPANY, ST. MAURICE DEPARTMENT 1805-1811}

\begin{tabular}{|c|c|c|c|c|c|c|}
\hline Fourrures & $1805-1806$ & $1806-1807$ & $1807-1808$ & 1808-1809 & $1809-1810$ & $1810-1811$ \\
\hline Castor (livres) & 138 & 170 & (170) & 170 & 180 & \\
\hline Rat musqué & 10,5 & 12 & 15 & 18 & & \\
\hline Loutre & 360 & 210 & & 190 & & \\
\hline Martre & 40 & 36 & & 36 & & 30 \\
\hline Vison & 30 & 30 & & 29 & & \\
\hline Lynx & 60 & 60 & & & & \\
\hline Ours & 300 & 480 & & & & \\
\hline Renard blanc & 180 & & & & & \\
\hline Pécan & & 60 & & & & \\
\hline Carcajou & & 30 & & & & \\
\hline Castoréum (livre) & & & & 90 & & \\
\hline
\end{tabular}

Cependant, ce nombre de paquets équivaut aussi à la cinquantaine de ballots que les frères Giasson recueillaient annuellement en HauteMauricie à la fin des années $1770^{44}$; pourtant, ces derniers étaient sortis pratiquement ruinés de leur expérience commerciale en HauteMauricie. Évidemment, pour de petits exploitants comme les Giasson, qui pratiquaient un commerce à petite échelle, quelques dizaines de ballots de fourrures accumulés ne parvenaient pas nécessairement à couvrir les coûts d'exploitation. Par contre, pour des compagnies comme la North West Company et la Hudson's Bay Company qui fonctionnaient à grande échelle, ce qui leur permettait notamment de réduire le coût d'achat des articles de traite et ainsi d'obtenir un retour plus substantiel par rapport au prix offert pour les fourrures, les mêmes dizaines de ballots rapportaient finalement davantage ${ }^{45}$.

44. Claude Gélinas, Haldimand..., op. cit.

45. Il reste que pour comparer adéquatement la rentabilité du commerce des Giasson par rapport à celui de la North West Company ou de la Hudson's Bay Company, il faudrait disposer d'informations sur le coût des marchandises ainsi que sur le coût de revient des fourrures amassées, ce qui n'est malheureusement pas le cas ici. 
TABLEAU 3

\section{ÉVOLUTION DES ENTRÉES DE FOURRURES DANS LES POSTES \\ DE LA NORTH WEST COMPANY, ST. MAURICE DEPARTMENT \\ 1805-1813}

$\begin{array}{lcrrrrrr}\text { Fourrures } & \mathbf{1 8 0 5}- & \mathbf{1 8 0 6}- & \mathbf{1 8 0 7}- & \mathbf{1 8 0 8}- & \mathbf{1 8 0 9}- & \mathbf{1 8 1 0}- & \mathbf{1 8 1 1 -} \\ & \mathbf{1 8 0 6} & \mathbf{1 8 0 7} & \mathbf{1 8 0 8} & \mathbf{1 8 0 9} & \mathbf{1 8 1 0} & \mathbf{1 8 1 1} & \mathbf{1 8 1 2} \\ \text { Castor (livres) } & 2993 & 3398,5 & 1629 & 2026 & 720 & & \\ \text { Rat musqué } & 3179 & 4188 & 4376 & 3008 & & & \\ \text { Loutre } & & 181 & & 82 & & & \\ \text { Martre } & & 189 & & 100 & & 99 & \\ \text { Vison } & & 45 & & 35 & & & \\ \text { Lynx } & & 170 & & & & & \\ \text { Ours } & & 45 & & & & & \\ \text { Pécan } & & 5 & & & & & \\ \text { Carcajou } & & 3 & & & & & \\ \text { Nombre } & & & & & & & \end{array}$

Source: ASQ, North West Company Account Book, 1804-1813.

De plus, ces comparaisons supposent que le poids et la valeur des paquets aient été les mêmes entre 1780 et 1867 . Pour ce qui est du poids, puisque le transport des ballots entre les Hauts-mauriciens et la vallée laurentienne s'est toujours effectué par canot durant cette période, et que le poids des ballots devait nécessairement être limité pour faciliter la tâche des voyageurs lors des nombreux portages, on peut présumer que ceux-ci ont toujours pesé en moyenne aux environs de 80 à 90 livres $^{46}$. Par contre, en ce qui concerne la valeur des paquets, on soupçonne qu'elle a nécessairement varié tout au long de cette période, compte tenu de la diversité des fourrures échangées et des fluctuations de leur valeur sur les marchés. Si on ignore la composition des paquets des Giasson, on sait que quelques années auparavant (vers 1765), le total des peaux échangées par les autochtones de la Mauricie avec le marchand Aaron Hart de Trois-Rivières comprenait $77,5 \%$ de peaux de castor, et $22,5 \%$ de pelleteries (peaux de

46. J. E. Lunn, Développement économique de la Nouvelle-France, 1713-1760 (Montréal, Presses de 1'Université de Montréal, 1986), 78; L.-R. Masson, op. cit., 1 : 51 ; J. W. Anderson, Fur Trader's Story (Toronto, The Ryerson Press, 1961), 115. 
martre et de loutre) ${ }^{47}$. De même, à l'instar de l'ensemble de l'Est canadien au XVIII ${ }^{\mathrm{e}}$ siècle, la Mauricie semblait constituer une région où les castors étaient particulièrement abondants ${ }^{48}$.

Cette situation n'avait vraisemblablement pas changé au temps de la North West Company et de la Hudson's Bay Company, du moins si l'on se fie à l'évolution des entrées de peaux de castor dans les postes de la Mauricie tout au long du XIX ${ }^{\mathrm{e}}$ siècle (tableau 4). D'ailleurs, la moyenne annuelle des entrées de peaux de castor dans les postes de la North West Company, entre 1805 et 1810, se rapproche de celle observée dans les années 1860 et 1870, alors que la Hudson's Bay Company payait pourtant environ moitié moins cher pour ce type de peau. Par contre, la même compagnie recueillait à cette époque beaucoup plus de pelleteries (peaux de loutre, martre, vison, rat musqué, etc.) que ne l'avait fait la North West Company au début du siècle. Comme ces pelleteries étaient généralement plus en demande que les peaux de castor dans la seconde moitié du XIX siècle, on peut présumer qu'au total, un ballot de la Hudson's Bay Company, dans les années 1860 et 1870, valait davantage qu'un ballot de la North West Company vers $1806-1807^{49}$. Bref, la North West Company a peut-être réussi, grâce surtout aux entrées de peaux de castor, à faire un commerce profitable en Mauricie avant 1807, mais elle n'était vraisemblablement pas parvenue, tout comme la Hudson's Bay Company avant les années 1860, à tirer du commerce local tous les revenus possibles; à cet effet, il suffit de comparer le total des peaux obtenues en 1806-1807 $(\mathrm{n}=8225)$ avec les totaux annuels moyens obtenus dans les années $1860(\mathrm{n}=11749)$ et 1870 $(\mathrm{n}=12275)$ et ce, auprès d'une population autochtone qui a toujours tourné autour de 200 individus.

Pour bien comprendre pourquoi le commerce des fourrures en Mauricie ne semble avoir atteint son apogée que dans la seconde moitié du XIX ${ }^{\mathrm{e}}$ siècle, il convient de s'attarder ici sur ceux qui fournissaient les fourrures aux commerçants, à savoir les autochtones de la région. En effet, l'étude de la participation des Atikamekw à ce

47. Exact List of the testes de boules Indians who are indepted to Mr Aaron Hart. ASTR, Fonds Aaron Hart, sans date.

48. Thomas Wien, «Castor, peaux, et pelleteries dans le commerce canadien des fourrures, 1720-1790», dans Bruce G. Trigger et al., dir., «Le castor fait tout». Selected Papers of the Fifth North American Fur Trade Conference, 1985 (Montréal, The Lake St. Louis Historical Society, 1987), 85; Toby Morantz, An Ethnohistoric Study of Eastern James Bay Cree Social Organization, 1700-1850 (Ottawa, Musées nationaux du Canada, Musée national de l'Homme, $1983), 18,38$.

49. La Hudson's Bay Company offrait environ $60 \%$ de plus pour les pelleteries dans les années 1860-1870 que la North West Company dans les années 1800. 
TABLEAU 4

\section{QUANTITÉS DE FOURRURES ÉCHANGÉES EN MOYENNE PAR ANNÉE DURANT CERTAINES DÉCENNIES DU XIX SIÈCLE}

\begin{tabular}{|c|c|c|c|c|c|c|c|}
\hline Fourrures & 1800 & & $330 *$ & 1840 & 1850 & 1860 & 1870 \\
\hline Castor (livres) & 2153 & 1051 & [769] & 486 & 640 & 2126 & 3749 \\
\hline Rat musqué & 3688 & 8993 & [7080] & 5166 & 5271 & 8455 & 7001 \\
\hline Loutre & 132 & 131 & {$[140]$} & 149 & 172 & 241 & 188 \\
\hline Martre & 145 & 500 & [619] & 738 & 469 & 818 & 1209 \\
\hline Vison & 40 & 124 & [137] & 150 & 194 & 290 & 1281 \\
\hline Lynx & 170 & 29 & [81] & 133 & 115 & 109 & 128 \\
\hline Total & 6328 & 10828 & [8825] & 6822 & 6861 & 12039 & 13556 \\
\hline
\end{tabular}

* Les données pour la décennie des années 1830 méritent d'être nuancées puisqu'elles ne sont disponibles que pour les années 1831-1832 et 1833-1834. Or, j’ai démontré ailleurs que les entrées de fourrures au poste de Weymontachie durant ces années étaient semblables à celles qui prévalaient durant les années 1820, dans le contexte de concurrence entre la Hudson's Bay Company et la King's Posts Company. Claude Gélinas, «Atikamekw Economy in the 1830's and the Hudson's Bay Company Account Books as Sources for Historical Ethnographic Studies », manuscrit soumis pour publication. Par contre, à compter de 1836, le volume des fourrures échangées au même endroit était similaire à celui qui prévalait durant les années 1840 . Si la situation à Weymontachie était représentative de ce qui prévalait à l'échelle du St. Maurice District, les chiffres entre crochets, qui représentent une moyenne entre le volume des entrées de la première moitié des années 1830 et celui des années 1840, sont probablement plus représentatifs de l'ensemble de la décennie 1830-1839. Par ailleurs, bien que les données exactes manquent pour les années 1820 , on peut s'attendre à ce que le nombre de peaux échangées en moyenne chaque année en Mauricie ait été semblable à celui observé au début des années 1830 .

Source: ASQ, North West Company Account Book, 1804-1813; HBCA, B. 230/d/16, 21, 31, 35,43 ; B. $134 / \mathrm{h} / 1$.

commerce, au cours du XIX ${ }^{\mathrm{e}}$ siècle, a permis de constater qu'entre les années 1820 et 1850, ce domaine économique occupait chez eux une place relativement secondaire ${ }^{50}$, à l'exception d'une période de quelques années, du mitan des années 1820 au début des années 1830 , où la concurrence entre la Hudson's Bay Company et la King's Posts

50. Claude Gélinas, «L'économie des gens de Weymontachie dans les années 1820, vue à travers les livres de comptabilité des compagnies de traite», Recherches amérindiennes au Québec, 28,2: 77; id., La gestion de l'Étranger. Les Atikamekw et la présence eurocanadienne en Haute-Mauricie, 1760-1870 (Sillery, Septentrion, sous presse); id., " Atikamekw Economy in the 1830's and the Hudson's Bay Company Account Books as Sources for Historical Ethnographic Studies », Manuscrit soumis pour publication. 
Company en Mauricie a favorisé une participation plus intensive des autochtones au commerce ${ }^{51}$. Dans l'ensemble, l'économie domestique des Atikamekw était basée sur la chasse au gros gibier qui fournissait, entre autres, les peaux pour se vêtir et la viande pour se nourrir. À cet égard, la faible quantité de vêtements achetés dans les postes de traite à cette époque, tout comme le fait que la nourriture - essentiellement de la farine - obtenue en échange de fourrures comblait moins de $2 \%$ des besoins caloriques des Atikamekw témoignent de la relative indépendance de ceux-ci face aux commerçants ${ }^{52}$.

Néanmoins, parallèlement à la poursuite du gros gibier, les Atikamekw piégeaient une quantité relativement modeste d'animaux à fourrure dont les peaux étaient échangées, principalement, contre des articles tels que des fusils et des munitions, des haches, des couteaux, des marmites, de l'alcool et du tabac, autant de produits qui facilitaient et agrémentaient la pratique du mode de vie traditionnel. De même, en comparant le volume des entrées de fourrures dans les postes de la North West Company entre 1805 et 1812 avec celui qui prévalait dans les postes de la Hudson's Bay Company dans les années 1830 à 1850 (tableau 4) - tout en tenant compte que les prix offerts pour les peaux sont demeurés relativement stables durant toute la première moitié du $\mathrm{XIX}^{\mathrm{e}}$ siècle - on peut émettre l'hypothèse que cette participation modérée des Atikamekw au commerce des fourrures a prévalu également durant les années 1800 et 1810 .

Par contre, plusieurs indices portent à croire qu'à compter de la fin des années 1850 , il y eut une diminution très prononcée de la population d'orignaux en Mauricie. Par exemple, les cas de famine ont augmenté soudainement chez les Atikamekw ${ }^{53}$; ceux-ci ont dû instaurer un droit de propriété individuelle sur les orignaux ${ }^{54}$, se résoudre à acheter dans les postes de traite des articles faits en cuir (mocassins, courroies de portage) ou des vêtements (chapeaux, pantalons) pour remplacer ceux qu'ils avaient coutume de confectionner eux-mêmes en peau d'orignal, et accepter d'augmenter considérablement leurs achats de produits alimentaires ${ }^{55}$. En d'autres termes, privés d'une importante source de viande et de cuir, les Atikamekw résolurent de

51. Claude Gélinas, «La traite des fourrures...», loc. cit.

52. Claude Gélinas, «L'économie des gens...», loc. cit.

53. HBCA, Weymontachingue Post Journal, 1857-1858, B. 230/a/3, fos. 6, 8, 10; $1864-$ 1866, B. 230/a/4, fos. 24-25, 28; Montreal Correspondence Inward, 1856, 1861, B. 134/c/73, fo. 289 ; B. $134 / \mathrm{c} / 83$, fo. 155 .

54. Archives provinciales des Oblats de Marie-Immaculée (Montréal), Lettre du missionnaire Andrieux au Révérend Père, Maniwaki, 1856?. Dossier Maniwaki, 2D9-9-13.

55. Claude Gélinas, La gestion de l'Étranger..., op. cit. 
se tourner vers le commerce avec la Hudson's Bay Company pour se procurer, notamment, de la farine de même que des vêtements et tissus en grande quantité.

Or, puisque ces articles étaient alors vendus à fort prix, il fallait fournir beaucoup de fourrures pour se les procurer, d'où l'augmentation sensible des entrées de peaux dans les postes de la Mauricie à compter des années 1860. La hausse des prix offerts pour la plupart des peaux à la même époque, laquelle fut d'ailleurs généralisée à l'ensemble du Canada, a pu encourager les Atikamekw à poursuivre leur virage vers une économie axée sur le piégeage intensif, mais elle n'en a sûrement pas constitué l'élément déclencheur.

\section{CONCLUSION}

L'histoire de la North West Company en Mauricie peut être divisée en deux grandes périodes: celle qui s'étend des environs de 1799 à 1807, durant laquelle la compagnie dirigea dans la région un commerce des fourrures que l'on pourrait qualifier de classique et qui s'avéra peut-être lucratif; et celle qui s'étend de 1807 à 1814, durant laquelle la North West Company, qui dans l'ensemble connaissait de sérieux problèmes financiers, a vu ses revenus en Mauricie diminuer considérablement. Toutefois, cette baisse des revenus aurait été en quelque sorte provoquée par les dirigeants de la compagnie qui, nouvellement locataires du Domaine du Roi depuis 1802, ne voulaient pas voir les postes mauriciens attirer la même clientèle que celle des King's Posts, ce qui contribuait à gonfler inutilement les coûts d'exploitation. Le choix fut alors fait de transformer les postes de la Mauricie en simples lieux de surveillance de la frontière des King's Posts, tout en faisant en sorte que le commerce y soit limité au minimum, au profit des établissements du Domaine.

Par ailleurs, l'analyse des données contenues dans les livres de comptabilité de la North West Company a fait ressortir l'influence exercée par les autochtones sur la rentabilité du commerce en Mauricie dans les premières décennies du XIX ${ }^{\mathrm{e}}$ siècle. Certes, la petitesse relative des revenus générés par le St. Maurice Department, du moins en comparaison avec ceux des postes voisins, s'explique en partie par le nombre somme toute peu élevé de chasseurs autochtones dans cette région (peut-être moins d'une quarantaine). Mais elle s'explique également par le fait que ces mêmes chasseurs ne souhaitaient probablement pas participer assidûment au commerce des fourrures. Comme ce fut le cas jusqu'au mitan du XIX ${ }^{\mathrm{e}}$ siècle, les autochtones de la Mauricie, au temps de la North West Company, ne voyaient dans la traite des fourrures qu'une manière avantageuse de se procurer une gamme 
limitée d'articles de traite, lesquels facilitaient et agrémentaient la pratique de leur mode de subsistance traditionnel et ce, en échange de fourrures qui étaient aisément obtenues à l'intérieur du mode d'exploitation du gros gibier.

Éventuellement, il sera intéressant de voir ce que révéleront les données concernant le commerce effectué par la North West Company dans les secteurs de Grand River, du Témiscamingue et des King's Posts - données qui sont d'ailleurs beaucoup plus abondantes que celles relatives à celui du Saint-Maurice - et de comparer les résultats avec ce que l'on connaît maintenant des activités de la compagnie en Mauricie. Lorsque toutes ces études pourront être combinées, un pas important aura été fait dans la documentation de l'histoire des « Nor'Westers» dans l'Est canadien en vue d'une meilleure compréhension de l'importance historique de cette compagnie à une échelle vraiment pancanadienne. 
